\title{
A Research on QoS Optimization in 4G Cellular Networks
}

\author{
Vandana Khare, D. Srinivasa Rao
}

\begin{abstract}
- cellular system conveys sight and sound website online traffic productively during ongoing transmission. in view that this device transmits blended media information, there may be a prerequisite to adjust the management price as indicated via the gadget situations an excellent manner to hold up fruitful device hobby. in the end, there is a need to allot the assets capably. Nature of provider $(\mathrm{QoS})$ improvement within the sort of system is additionally trying out specially in $4 G$ cellular systems. severa plans were proposed for QoS development in cellular structures which be a part of name Admission control (CAC) and electricity control Scheme. Be that as it is able to, CAC with electricity control isn't always sufficient to offer the most ideal asset use. alongside the ones lines, it is straightforward to build up an powerful QoS primarily based definitely resource Allocation and Scheduling Scheme for normal sight and sound site visitors. The primary objective of proposed studies paintings is to build up a nice of provider based resource Allocation and Adaptive rate Scheduling (QRAAS) plot in cellular systems to improve prepare dependability, boom the framework limit and enhance framework proficiency and therefore to improve best of service in cell systems. To execute the formerly stated QRAAS conspire, resource Allocation based definitely call Admission manage (RACAC) plan and Adaptive price Scheduling (ARS) plot were created.
\end{abstract}

Watchwords: ARS, CAC, QoS, QRAAS, RACAC

\section{PRESENTATION}

Mobile 4G systems will supplant the modern cell cellular telephone arranges in an IP primarily based completely device. With appearance of IPv6, every tool on the earth can without a good deal of a stretch get a definitely one among a type IP address. This allows full IP based interchanges via a mobile cellular phone. The QoS is the capability to provide particular need to at least one-of-a-kind programs, customers, or records streams, or to make sure a particular diploma of execution to an facts circulate $[1,3]$. as an example, a required piece fee, dispose of, jitter, package dropping risk and bit mistake rate might be ensured. The QoS certifications are large if the tool limit is insufficient, particularly for ongoing gushing media applications, as an example, voice over IP, net primarily based completely video games and IP Telephony, because the ones regularly require fixed piece fee and are postpone sensitive, and in systems in which the limit is a confined asset as we see in cellular information correspondence.

OFDMA is a very mainstream law method which uses increasingly sort of channels whose transmission capacity is

Revised Version Manuscript Received on August 19, 2019.

Dr. Vandana Khare, Professor -ECE department, CMR College of Engineering \& Technology, Hyderabad, Telangana, India(Email: : vandanakhare2018@gmail.com)

Dr. D. Srinivasa Rao Professor in ECE Department, JNTU College of Engineering, JNTUH,Hyderabad, India(Email: : dsraoece@gmail.com) better than that of the facts this is to be moved. OFDMA constantly underpins higher records rate administrations. however, some other Medium get entry to manipulate conference (MAC) is critical to cope with parcel get to capably OFDMA faraway systems [2]. in an effort to make use of such arranges, efficient call Admission manipulate (CAC) and Medium get right of entry to control (MAC) conventions are simple for the QoS provisioning in $4 \mathrm{G}$ scenario. Be that as it could, CAC isn't completely first-rate to offer the most best asset makes use of [3] due to the bursty idea of package visitors. In an integrated multiclass packetized set up, resource Allocation with energy imperatives is wanted for proscribing the obstruction and enhancing device execution to enhance first-class of carrier in $4 \mathrm{G}$ cellular system [4].

on this paper, we proposed another useful resource Allocation primarily based name Admission manage (RACAC) calculation for numerous administrations like voice, video and facts for multiclass clients. It involves a selection the maximum best association of suitable customers with maximum ideal price, to lower the impedance degree and talk to blockading probability at the same time as the Adaptive charge Scheduling calculation modifies the price for each session. The remainder of the paper consists as pursues: segment II offers the trouble proclamation, location III gives insights concerning the associated work. the general work plan approximately proposed philosophy is delineated in segment IV. subsequent, phase $\mathrm{V}$ offers the exhibition assessment for the proposed methodology eventually, ends are given in area VI.

\section{DIFFICULTY ASSERTION}

it's miles a profoundly scary task to enhance unwavering exceptional, skills and limit close to ensured satisfactory of service in $4 \mathrm{G}$ mobile structures. the key idea is to accumulate a QoS primarily based useful resource Allocation, effective power control and Scheduling (QRAAS) Scheme that improves set up dependability, effectiveness and in cutting-edge organize restriction through gifted asset distribution and legitimate asset utilization for maintaining up the required great of carrier in cellular structures.

A QoS improvement in $4 \mathrm{G}$ system is based upon on aid Allocation with name Admission manipulate (RACAC) plan to decorate put together dependability. An Adaptive price Scheduling (ARS) plot utilising Heuristic based comments control Unit (FCU) reason improves framework productivity. 


\section{RELATED PAINTINGS}

BijanGolkar[7] has created Allocation of assets in self-coordinated versatile structures and proposed a community Clustering Scheme in which route of motion of cells is portrayed by the use of a meeting of towers or Base Stations (BS). As consistent with the proposed plan, potential consequences emerge. either each flexible purchaser speaks with just a unmarried BS or with multiple BS in a booking cellular.

as the challenge of property over the making plans cell is appropriated, each booking cellular is evidently related without getting facts from past level. Be that as it could, strength management is an excessive undertaking in such type of self-walking systems.

MohamedKhadim[8]hasanalyzed and looked at asset challenge approaches for new gushing administrations in cell and portable systems. In flexible structures, schooling of administrations are very well-known. In first class, there are Variable Bit price (VBR) and consistent Bit charge (CBR) and inside the special kind, gushing call (video, voice, communicate) is given, this is implemented in contemporary age dependent on QoS and request of consumer. though, there may be cast off in the plan.

Magnus Protester [9] has proposed high growth based totally useful resource Allocation the use of Context-aware hypothesis. below this plan allotment of assets is based upon on the existing traffic and as indicated through the website online visitors, scheduler performs capacities: (1) Time openings are allocated beforehand of time for the upcoming traffic as in line with defer conditions (2) delay spending plan is set up for each coming close to solicitation with the help of application capability. within the proposed plan, Scheduler assumes a full-size challenge as throughput is predicted as a ways as variety productivity concerning framework restrict and inclusion place. here, the scheduler can growth the website site visitors load with out increasing the device multifaceted nature although utility capability can bolster frequently range of customers through the use of regular trade landings. however, the precept disadvantage of this plan is that the formally transmitted consultation can't be applied once more and the amount of cycles is stepped forward. Henceforth, legitimate resource utilization is beyond the arena of creativeness.

Jraifi Abdelouahed [10] has inspected troubles that limit execution of cell structures. additionally, a scientific technique for thinking about the co-divert obstruction in WCDMA framework has been created. A miniaturized scale zoning essential plan to determine the (S/I) share is embraced in this paper. This explanatory final effects is utilized to foresee the amount of customers steady with miniaturized scale zones. This final consequences is of essential significance because it contributes sincerely in enhancing the man or woman of administrations. besides the amount of clients ought to be controlled for upgraded QoS execution.

N. Mohan and T. Ravichandran [11] have expected to format each other CAC calculation with electricity manage for a few, administrations like voice, video and statistics for multiclass customers. It involves a choice the best arrangement of perfect customers with notable transmitting pressure diploma, to reduce the impedance stage and get in touch with quit rate. additionally, a flexible reserving plan is meant to allot maximum perfect rate for each site visitors line and to restrict the planning put off. The proposed calculations achieve less call blocking off opportunity and most best rate with decreased postponement. besides there was a deferral within the plan.

Salman A. AlQahtani et al [12] have proposed and broke down a effective uplink-making plans plan within the occasion of RAN sharing method. This new plan is known as the Multi-administrators Code department Generalized Processor sharing plan (M-CDGPS). It makes use of each bendy price designation to expand the asset use and GPS systems to offer lower priced administrations to each administrator. The exhibition exam of this plan is inferred using the GPS execution model. Likewise, it's far contrasted and static rate M-CDGPS plot. consequences reveal that the proposed bendy rate MCDGPS making plans plan improves every framework throughput and everyday postponements. anyway the hypothetical defer positive increments as the token field size increments.

Tajje-eddine Rachidi et al [13] have provided QaPC and QaHO devices, which rely upon the elegance of administration, the bit fee, and the carrier Degradation Descriptor (SDD) as empowering QoS parameters. The predicted QoS conscious device actually improves QoS agreement retaining for top class transportable clients, even as growing asset usage and enhancing SHO gathering. anyway there may be over-burden within the plan.

youthful long Chen et al [14] have prepared a propelled plan which consolidates the CAC and power manipulate components and works in a focused control mode. The embodiment of the predicted focused call confirmation manipulate (CCAC) plot is to enroll in the two instruments and to deal with the call affirmation choice as an eigen-decay problem. with a view to lower the computational multifaceted nature of the eigen-disintegration issue, the paper proposes an extra plot, which makes use of a famous ability in place of direct estimation. The proposed plan, in spite of the same old guess, outflanks unsurprising name confirmation strategies regarding its blocking off price and blackout fee. therefore, the actual SIR of every connection in a neighboring base station may not make sure with the outcome that blackout may additionally occur.

Peifang Zhang and Scott Jordan [15] have advanced a form for Connection Acc.

\section{PROPOSED QOS IMPROVEMENT SCHEMES}

\subsection{Aid Allocation Scheme}

In useful resource Allocation based name Admission manipulate (RACAC) calculation, the device chooses whether or not or now not to concede the new durations or not and to set the Transmission price for those conceded periods. Disconnected alternatives depend on the beyond, gift and future channel situations, landings and flights. therefore, on line picks are utilized as they recall virtually the over a substantial span of time information.

allow TPi be the objective throughput of the imminent clients within the device.allow ATPi be the performed

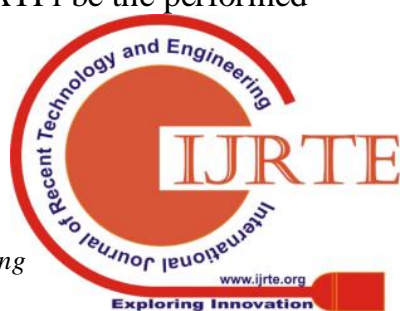


throughput of the present day and sparkling advent clients within the device.

provide Si a danger to be the passage time of the session I sooner or later of interest of the device.

deliver ri a risk to be the rate appointed to consultation I sooner or later of interest of the gadget.

Operational Steps

level 1: whilst every other customer touches base for a session I, record the access.

stage 2: On patron's touchdown, goal throughput TPi is relegated, which relies upon on no. Of customers and relegated information rate inside the machine.

stage three: executed throughput ATPi is determined via the accompanying situation

The touchdown time of the client I is supposed by using manner of Si. Age of the client I at time $t$ is given via

$$
\operatorname{Age}_{i}(t)=t-S_{i}
$$

Achieved throughput of user $i$ from entry time $S_{i}$ until time $t$ is given by equation 4.2 [ref.13]-

$$
x_{i}(t)=\frac{\left[\sum_{k=s_{i}}^{t} r_{i}(k)\right]}{\operatorname{Ag}_{i}(t)}
$$
accompanying situations for ATPi-

1.while done throughput isn't always precisely the goal throughput, allot a fee boom and compute ATPi by using way of the given equation.

2.whilst achieved throughput is more brilliant than the goal throughput, dole out a charge decrement and parent ATPi by means of way of the given recipe.

3.when both the situations do now not emerge, i.E., finished throughput is equal to purpose throughput, all calls can be conceded, real to shape

\subsection{Adaptive Rate Scheduling Scheme-}

at some stage in the segment of the consultation, the charge Rallocate is dispensed for drawing near customers. From that point onward, the Time location time period $\mathrm{T}$ and $\mathrm{Q}$ size of consultation I, are appointed and the touchdown fee rest-get admission to is evaluated. at the factor at the same time as client touches base at the base Station, the price is balanced dependent on $\mathrm{fb}$ have been given from as of now conceded session. concurrently, FCU makes a selection the postponement for limit diploma Dthreshold. It likewise evaluates the average Scheduling put off Davg(i) from first client and assessments the favored situations. on the off threat that Davg isn't always precisely or same to Dthreshold, FCU pre-empts all the customers.If Davg is extra than Dthreshold, mistake in touchdown fee estimation is shown. afterward, charge Rallocate is doled out and the landing rate for consumer is assessed. At lengthy final, assessed fee is despatched as feedback to Base Station (BS) in light of which, BS modifies the charge for coming close to purchaser.

let Rallocate be the disbursed price for the imminent session.

provide $\mathrm{T}$ a hazard to be the outlet span.

deliver $\mathrm{Q}$ a chance to be the line duration of the session.

Operational Steps

level 1: The landing rate Rest_arrival is assessed while a patron arrives. degree 4: After figuring of completed throughput, test the

stage 2: After estimation of landing fee, BS alters the price relying on comments obtained from as of now conceded customer

stage 3: feedback control Unit (FCU) makes a choice the postponement for limit diploma Dthreshold.

stage four: common Scheduling postpone Davg for consultation $\mathrm{I}$ is classified the use of the accompanying condition five. 1 [ref.88]-

The regular planning postponement of the substantial number of customers is classed from the maximum important session I conceded into the tool

$$
D_{\text {schedule }}(i)=\frac{Q_{\text {session }}(i)+T \cdot R_{\text {est arrival }}(i)}{R_{\text {allocated }}(i)}
$$

degree five: After remember number of average Scheduling postpone Davg, the accompanying conditions are checked-

1.while average Scheduling delay is not exactly or equal to Threshold put off, the remarks manipulate Unit allows to concede each one of the customers.

2.whilst average Scheduling put off is extra than the threshold postpone, at that aspect,

a. Allocate rate Rallocate and gauge the touchdown charge for patron.

B.ship evaluated fee as comments to Base station.

C.Base station modifications the fee for coming close to patron.

\section{EXECUTION EVALUATION \& RESULTS}

on this segment, we recreate our proposed useful resource Allocation based completely call Admission manipulate conspire (RACAC) and Adaptive price Scheduling Scheme for actual-time traffic in WCDMA wireless Networks. The duplicate tool applied is NS-three, it truly is a appreciably beneficial interest device that gives discrete occasion reenactment of patron characterized structures. in the exercise, flexible hubs waft in a six hundred meter x 600 meter square district for fifty seconds reproduction time. Introductory regions and traits of the hubs are obtained using the arbitrary waypoint (RWP) model of NS-3. All hubs have a similar transmission scope of 250 meters. We consider our RACAC just as RACAC-ARS conspire towards the everyday call affirmation manage CCAC and CCACFS plans.

in the primary diploma, a situation, of 6 hubs with 6 base stations for the territory period 600x six hundred meter cross for Variable Bit rate (VBR) traffic, is made [45]. The information transmission fee at some stage in reenactment is 0. fifty eight Mbps, the amount of clients is 6 , the parcel length is 512 and the complete pastime time is 50 seconds.

\subsection{Based on number of clients -}

parent.5.1.1. Demonstrates that the quantity of clients is differed from 1 to six and Packet transport Ratio from 0 to at least one.2. as a result of effective asset distribution, plainly our RACAC Scheme has more conveyance percentage at the same time as contrasted and CCAC Scheme. 


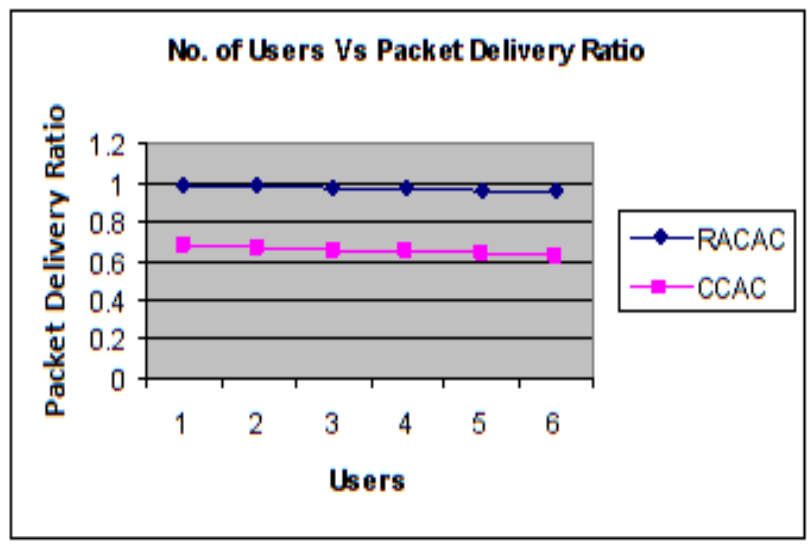

Fig 5.1.1: Users vs. Packet Delivery Ratio

determine.5.2.2. Demonstrates the deferral of website traffic happened whilst the amount of clients is changed for zero to 500 milliseconds delay. due to the fact clients depend upon their Adaptive fee, unmistakably the postponement associated with RACAC Scheme is lots less whilst contrasted with CCAC Scheme.

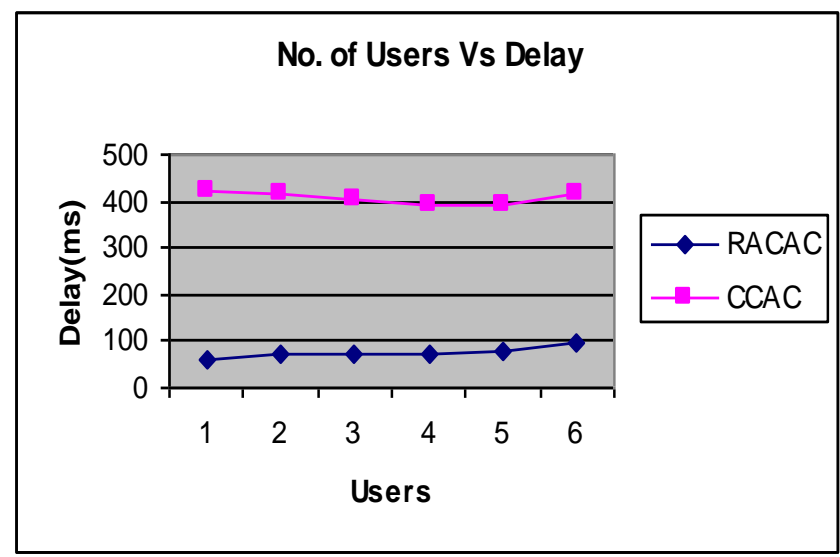

Fig 5.2.2: Users vs. Delay

From discern.5.3.Three, it can be found that because the variety of customers growth, RACAC Scheme attains higher throughput while as compared to CCAC Scheme. This is due to the fact completed throughput is constantly in comparison with purpose throughput and at the same time as determined to be a whole lot much less, a rate increment is carried out. Similarly, decrement is carried out even as wanted.

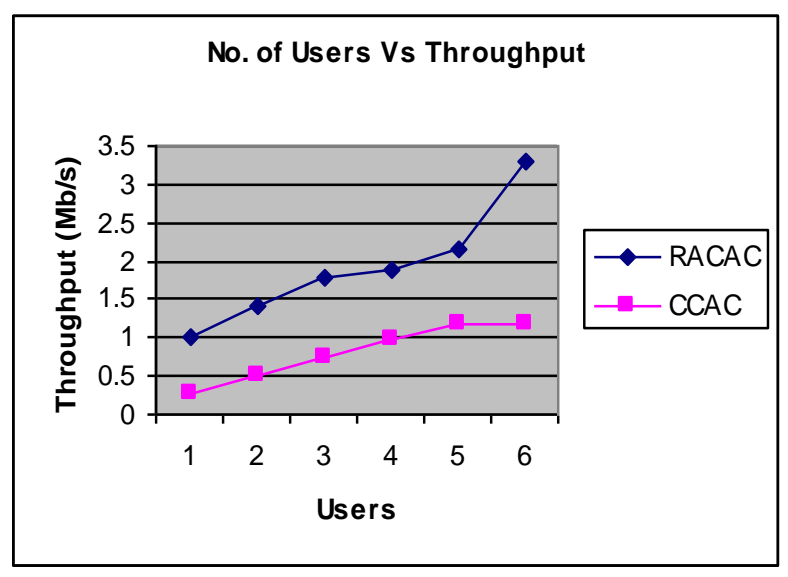

Fig 5.3.3: Users vs. Throughput

\subsection{Based on variety of customers}

RACAC-ARS machine is contrasted and Centralized name Admission manage and glued fee Scheduling (CCAC-FS) plot. due to suitable asset element, from decide five.2.1, obviously RACAC-ARS plan accomplishes higher conveyance percentage, at the same time as contrasted with the CCACFS conspire.

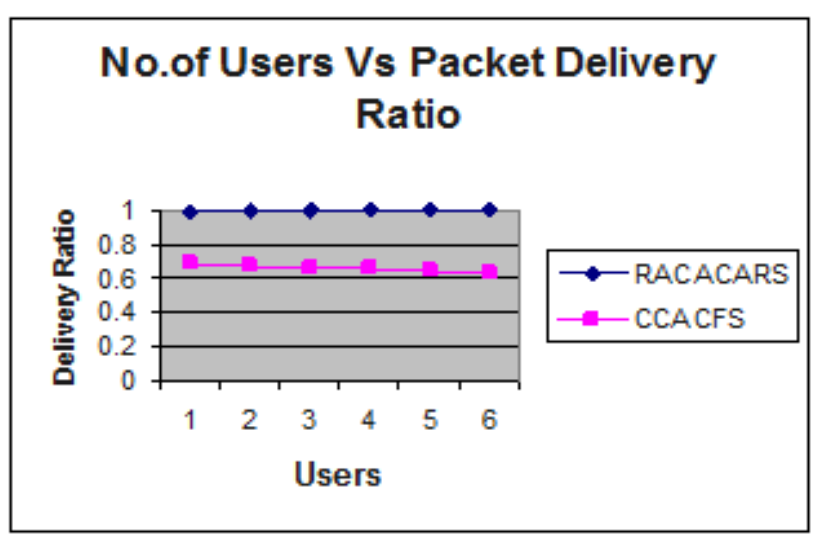

Figure 5.2.1: Users vs. Packet Delivery Ratio

Correspondingly, from the normal throughput regarded in discern 5.2.2, it thoroughly can be seen that RACAC-ARS plan achieves better throughput whilst contrasted with CCAC-FS conspire. that is considering that each time a customer arrives; the get admission to fee is evaluated and despatched as enter to the lowest station.

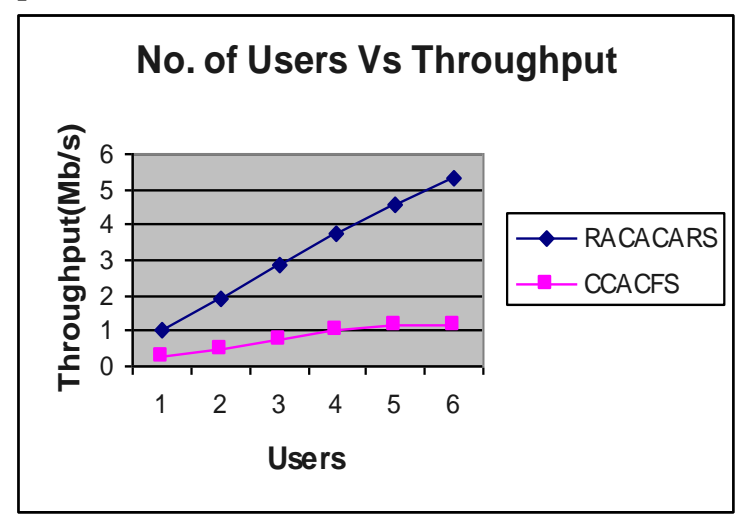

Figure 5.2.2: Users vs. throughput

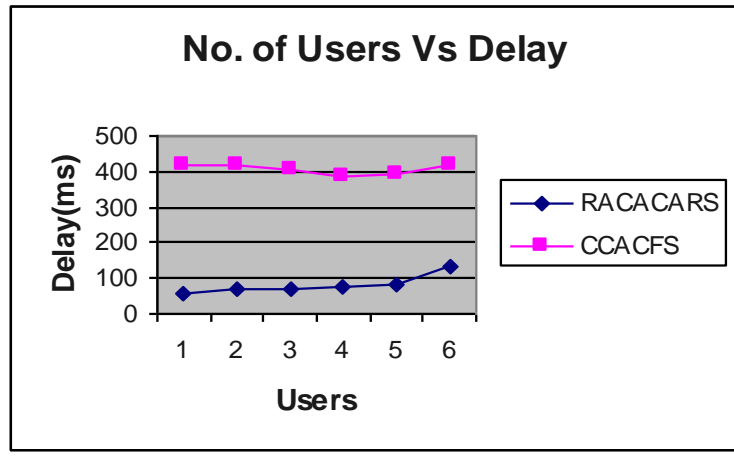

Figure 5.2.3: Users vs. Delay 
Likewise, from the regular deferral seemed in discern five.2.3, it has a tendency to be seen that RACAC-ARS plan accomplishes lesser put off when contrasted with CCAC-FS conspire. this could be confident with the aid of continuously contrasting the restriction put off and the normal postponement and in a while using the critical situations.

\section{STOP}

presently a day's $4 \mathrm{G}$ systems are frequent and it is with the aid of the usage of and via idea to be the up and coming age of transportable registering. Its usage and selections make it recognized from all exceptional buddy advancements. in an effort to keep its administrations accessible constantly, there's a want to improve its fine of provider with one-of-a-kind parameters.

on this proposed work, the created plan for resource Allocation with call Admission manipulate (RACAC) improves arrange unwavering high-quality with the aid of endeavoring the amount of periods that are obstructed due to insufficient belongings. The precept idea of asset allotment in the plan is applied to construct records price sooner or later of transmission as indicated by using consumer prerequisite and all of the at the same time as beautify throughput for non-forestall sight and sound site visitors utilising Variable Bit charge web site visitors for transmission. The aid Allocation with call Admission control plot for reducing side structures boosts training for clients whilst expanding statistics transmission rate. It likewise guarantees that another session does not disregard the QoS of continuous periods with the assist of Adaptive fee Scheduling (ARS) conspire. In ARS plot, Heuristic based totally method finds the arrangement close to the real one. The trendy method is based upon comments control Unit cause. alongside these lines, our proposed plan makes use of the assets successfully and expands otherworldly productivity of the system. As indicated thru stop result studies, it's far seen that our proposed plan improves throughput and decreases postpone for $4 \mathrm{G}$ cell Networks.

\section{REFERENCES}

1. VarshaJagiasi,Dr.NupurGiri,"ResourceAllocation techniques for cellular systems," worldwide magazine of Engineering research and generation, vol. five, no. 8, August. 2016.

2. AymenBelghith and NasrinTurki, "Adaptable name affirmation controlwith seizure in LTE systems," IEEE worldwide conference onCommunications (ICC), May2016.

3. Madhujain and Ragini Mittal, "flexible name affirmation control and resource Allocation in multi server far flung cell structures, vol. 12, no. 1, pp. 71-eighty, March 2016.

4. Mehmet Ozerk Memis,Ozgur Ercetin,,"useful resource component for measurable QoS guarantees in MIMO cell systems," EURASIP magazine on wireless Communications and networking,volume32,trouble 2, might also 2015.

5. G. Nantha Kumar and A. Arokiasamy , ",An efficient blended name Admission manage and Scheduling algorithm to enhance excellent of provider for Multimedia services Over next technology faraway structures "customary journal of pc packages, volume 107,No. 16, December 2014.

6. Juan Liu, Wei Chen,Member, IEEE, YingJun Zhang, Senior Member, IEEE, and Zhigang Cao, Senior Member,
IEEE ,An software Maximization Framework for honest and green Multicasting in Multicarrier a long way off cellular set up, IEEE/ACM TRANSACTIONS ON NETWORKING, VOL. 21, NO. 1, FEBRUARY 2013.

7. BijanGolkar,studentMember,IEEEandElvinoS.Sousa,Fell ow,IEEE,resource Allocation in autonomous cell systems, IEEE TRANSACTIONS ON wireless communication,typical FOR book in 2013.

8. Mohamed KadhemKarray, Yasir Khan (2012) "assessment and correlation of asset allotment structures for cutting-edge gushing administrations in remote mobile systems", IEEE TRANSACTION ON COMMUNICATIONS VOL12,NO.three JANUARY 2012.

9. Magnus Proebster, Matthias Kaschub, Thomas Werthmann1 and Stefan Valentin "placing conscious asset mission for mobile far off systems" EURASIP magazine on wireless Communications and Networking 2012.

10. Jraifi Abdelouahed, "A Theoretical Predictionof users in WCDMA Interference", journal of Theoretical and carried out data era thirty first January 2012. Vol. 35 No.2.

11. N. Mohan and T. Ravichandran, "An efficient Multiclass call Admission manipulate and Adaptive Scheduling For WCDMA wireless community",ecu journal of clinical research 33.four (2009).

12. Salman A. AlQahtani, Ashraf S. Mahmoud and Asrar U. Sheik, "Execution evaluation of Adaptive charge Scheduling Scheme for 3G WCDMA wireless Networks with Multi-directors", Communications, 2007. ICC'07. IEEE international convention on. IEEE, 2007.

13. Tajje-eddine Rachidi, Amal Yasmine Elbatji, Mehdi Sebbane and Hicham Bouzekri, "QoS-conscious power manage and Handoff Prioritization In 3g WCDMA Networks", wireless Communications and Networking convention, 2004. WCNC. 2004 IEEE. Vol. 2. IEEE, 2004.

14. younger-lengthy Chen, Jyu-Wei Wang, Jyh-Liang Chen and Jyh-Horng Wen, "Execution of call Admission control with strength control in Multimedia cell systems", worldwide magazine of implemented science and Engineering five.1 (2007).

15. Peifang Zhang and Scott Jordan, "pass Layer Dynamic useful resource Allocation with centered Throughput for WCDMA information", IEEE Transactions on wireless Communications, Vol. 7, No. 12, December 2008.

\section{AUTHORS PROFILE}

Dr. Vandana Khare is thru and thru functioning as a Professor at CMRCET, Hyderabad. She has gotten $\mathrm{PhD}$ (wireless conversation) from JNTU Hyderabad. She has over decades of involvement inside the field of teaching. Her commitments include round 35 research papers in diaries and conferences of countrywide/widespread notoriety. Her areas of intrigue contain media frameworks, correspondence and Networking and Biomedical sign coping with. She is a senior person from ISTE, IETE and IEEE specialized social orders.

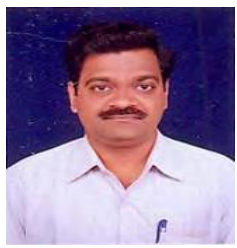

Dr. D. Srinivasa Rao received the B.Tech degree (ECE) from Nagarjuna University in1986, M.E (Digital Systems) from Osmania University in 1994 and $\mathrm{PhD}$ (Computer and Communication Networks) from University of Hyderabad in 2004. His research interest is in the area of communications and computer networks. Presently 12 research students are working under his guidance. He has 22 publications in various National, International Conferences and Journals. He has 27 years of teaching experience. He worked at CBIT as Lecturer in ECE Department for 6 years during 1988 1994. He worked at ECE Department of JNTU, Anantpur in various capacities for 11 years during 1994-2005.Presently he is working as

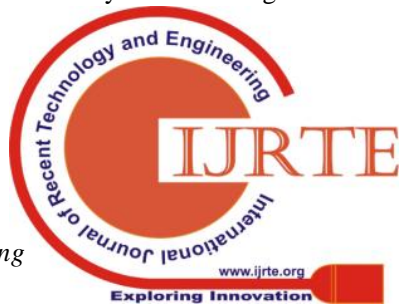


A Research on QoS Optimization in 4G Cellular Networks

Professor in ECE Dept. of JNTU CE, Hyderabad. He has chaired sessions at various national conferences. He is advisory committee member for GNIT, Hyderabad. He is also governing body member for Syed Hashim College of Science \& Technology, member of JNTU forum for Science \& Society and Coordinator for campus networking at JNTU CE, Hyderabad. 